\title{
Oil Movement Investigation at Pore-Scale in Waterflood Fluvial Reservoir
}

\author{
Fan Liu, ${ }^{1, a}$, Xuelin $\mathrm{Wu}^{2, \mathrm{~b}}$, Wensheng Zhou ${ }^{3, \mathrm{c}}$, Jian Shen ${ }^{1, \mathrm{~d}}$, Chen Liu ${ }^{1, \mathrm{e}}$ \\ ${ }^{1}$ CNOOC Research Institute, China \\ ${ }^{2}$ Research Institute of Petroleum Exploration \& Development, China \\ aliufan4@cnooc.com.cn, bwu-xuel@petrochina.com.cn, czhouwsh@cnooc.com.cn, \\ dshenjian@cnooc.com.cn, eliuchen4@cnooc.com.cn
}

\begin{abstract}
Keywords: fluvial reservoir; NMR; pore-scale residual oil distribution; horizontal well; Abstract. In the process of fluvial reservoirs development, bottom water coning leads to the problem of increasing water cut and low oil production. There are still significant amount of remaining oil in the upper layers of reservoirs after long-term waterflood. Drilling one or more lateral drainholes near the top of the formation is the main acceptable enhance oil recovery techniques to unlock additional recoverable reserves, but the microscopic oil movement in pores of different sizes is unclear. Nuclear magnetic resonance (NMR) is an effective way for analyzing pore structures and quantifying oil saturation in the rock matrix. In this paper, three-dimension physical model experiment was performed to evaluate the distribution and movement of oil in pores. During the water flooding progress, oil in large-medium pores was displaced first. After further displacement, oil in small pores begin to be produced. The oil recovery of small pores was even larger than the medium and large pores in the later stage of waterflood because of imbibition effect. The ultimate recovery of large pore in both top and bottom formation was almost the same, while the ultimate recovery of small pores varied with the degree of displacement. Substantial amount of remaining oil existed in the upper layer and in the small-medium pore of bottom layer after long-term waterflood.
\end{abstract}

\section{Introduction}

Fluvial reservoir is one of main reservoir types. The permeability of upper layers is usually low, and the permeability of bottom layers is usually high. After long-term waterflood, watered out will be the common problem. The injected water slumps downward in the permeable formation and sweeps only the lower part. The remaining oil is usually enriched in the top layers at high water-cut stage ${ }^{[1]}$.

Several experimental techniques have been proposed to describe the remaining oil distribution. Cast thin sections of a reservoir core is one of the traditional method ${ }^{[2]}$, but this method damages reservoir cores while obtaining slices. Another widely used experimental technique is to use a micro-visualized model instead of a reservoir core to simulate a displacement process as well as the distribution of remaining oil ${ }^{[3]}$. However, the influence of interstitial matter on the distribution of residual oil cannot be taken into consideration. X-ray computed tomography is often used to detect the rock matrix, but it is not sensitive to fluid changes in cores ${ }^{[4]}$. Numerical simulations require some assumptions to achieve mathematical completeness, and some of the parameters are uncertain ${ }^{[5]}$. NMR is an accurate, quick, non-destructive technology for core testing ${ }^{[6]}$. The signals in NMR measurement received only from fluids in pores. The hydrocarbons can be distinguished from brine which doped with paramagnetic ions because the signals from brine are shield, so that the signals only come from oil. NMR transverse relaxation time $\left(\mathrm{T}_{2}\right)$ represents the fluid content in the pores of different sizes. The shorter $\mathrm{T}_{2}$ relaxation time corresponds to the smaller pores, and vice versa ${ }^{[7]}$. Through the $T_{2}$ distribution analysis, the residual oil distributions in pores of different sizes are quantified, and the accurate oil saturation can be calculated to investigate the oil displacement process in pores of different sizes.

In our study, low-field NMR technology was used to evaluate the oil movement and residual oil distribution in pores of different sizes after horizontal producer development and gel treatment. The results could be used to provide theoretical basis for subsequent adjustment of long-term waterflood fluvial reservoirs. 


\section{NMR Technique Principle and Application}

NMR is a non-destructive analytical method to detect and character hydrocarbons in reservoir. NMR signals come from hydrogen atoms. More hydrogen atoms bring contribute to stronger signals ${ }^{[8]}$. Because the hydrogen atoms exist in both water and hydrocarbons, it is difficult to distinguish NMR signals from water and oil phases (especially light oil). In order to separate oil-phase signals from mixed signals, the core slugs are doped in a paramagnetic solution ${ }^{[9]}$. Paramagnetic ions are able to diffuse into the core samples. NMR $\mathrm{T}_{2}$ signals from the water phase reduce below the dead time of NMR, while the signals from oil remain detectable without any loss. In this experiment, Additional $\mathrm{Mn}^{2+}$ ions in the brines were paramagnetic ions.

Previous studies showed that the relation between the NMR $\mathrm{T}_{2}$ relaxation time and pore sizes is as follow ${ }^{[10]}$ :

$$
\frac{1}{T_{2}}=\rho \frac{s}{V}
$$

where $\mathrm{S} / \mathrm{V}$ is the specific pore surface area per volume which is inversely correlated with the pore diameter, $\mu \mathrm{m} ; \mathrm{T}_{2}$ is transverse relaxation time, $\mathrm{ms} ; \rho$ is interfacial relaxivity determined by the mineral constituents and surficial properties of the pores, $\mathrm{ms} / \mu \mathrm{m}$. In this experiment, when $\rho$ is 1.71 $\mathrm{ms} / \mu \mathrm{m}$, the pore radius distribution curve and conventional mercury curve fit well.

According to the analysis, the small pores (less than $2 \mu \mathrm{m}$ ) were defined as the pores with $\mathrm{T}_{2}$ less than $10 \mathrm{~ms}$, the medium pores $(2-20 \mu \mathrm{m})$ were defined as the pores with $\mathrm{T}_{2}$ between $10 \mathrm{~ms}$ and 100 $\mathrm{ms}$, and the large pores (more than $20 \mu \mathrm{m}$ ) were defined as the pores with $\mathrm{T}_{2}$ longer than $100 \mathrm{~ms}$.

\section{Horizontal Producer Experiment using NMR}

This experiment was used to study the oil displacement process and residual oil distribution of fluvial positive rhythm reservoir at pore scale after long-term waterflood with horizontal well.

Laboratorial Model and Fluid Properties. A three-dimensional physical model was generated by cemented sand. The model based on the characteristics of positive rhythm reservoir. Figure 1 shows the schematic diagram of the plate model. The model was $30 \mathrm{~cm} * 26 \mathrm{~cm} * 4.5 \mathrm{~cm}$, wrapping by epoxy resin outside. It was composed of three layers. Each layer was $1.5 \mathrm{~cm}$ in thickness. The model was vertically heterogeneous and horizontally homogeneous. One horizontal producer, two vertical producers and two vertical injectors were fixed in the model. The vertical producers penetrated all three layers and the horizontal well was in the middle of top layer. The oil was $26 \mathrm{mPa} \cdot \mathrm{s}$ in viscosity and $0.88 \mathrm{~g} / \mathrm{cm}^{3}$ in density $\left(20^{\circ} \mathrm{C}\right)$, which was same to the viscosity and density of reservoir crude oil in M-1 block. The brines used here was $\mathrm{MnCl}_{2}$ solution with $\mathrm{Mn}^{2+}$ concentration was $15000 \mathrm{mg} / \mathrm{L}$. It could kill the water signal so that only oil can be detected.

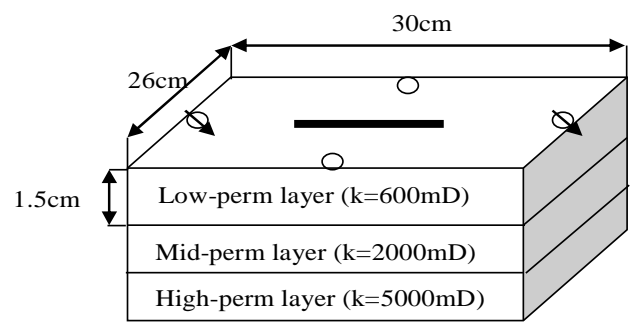

Fig.1 Schematic of Model and Wells Position

Equipment and Procedures. The main components of the apparatus included plastic pipelines, two constant-flux pump, fluid accumulator, a backpressure regulator, NMR analyzer, etc.

1. Preparing and pretreating physical model.

2. The model was weighed dry, and was saturated with prepared brines under vacuum. Aging under static status for 3 days.

3. The model was saturated with prepared oil, and aging under static status for 3 days. 
4. Shut horizontal well, open vertical wells. Injecting prepared $\mathrm{MnCl}_{2}$ solution at a flow rate of $0.5 \mathrm{~mL} / \mathrm{min}$, until water cut reached $95 \%$.

5. Shut off two vertical producers, open horizontal producer. Injecting prepared $\mathrm{MnCl}_{2}$ solution at a flow rate of $0.5 \mathrm{~mL} / \mathrm{min}$, until water cut reached $95 \%$.

6. In procedure 4-5, NMR analyzer was used to measure the transverse relaxation time $\left(\mathrm{T}_{2}\right)$ for each flooding state.

Results and Analysis. Figure 2 shows the NMR $\mathrm{T}_{2}$ distribution results. Because the water signal is "killed" by $\mathrm{MnCl}_{2}$, the curve envelope area only indicates the oil saturation. We can see that the oil saturation was still comparatively high after vertical well waterflood $\left(f_{w}=95 \%\right)$. Moreover, the displacement rate of movable oil gets slower over time. After shutting off vertical well and opening horizontal well, the oil saturation reduced very fast at first. Compare with vertical well development, horizontal well has more production during high water-cut stage $\left(f_{\mathrm{w}}>80 \%\right)$.
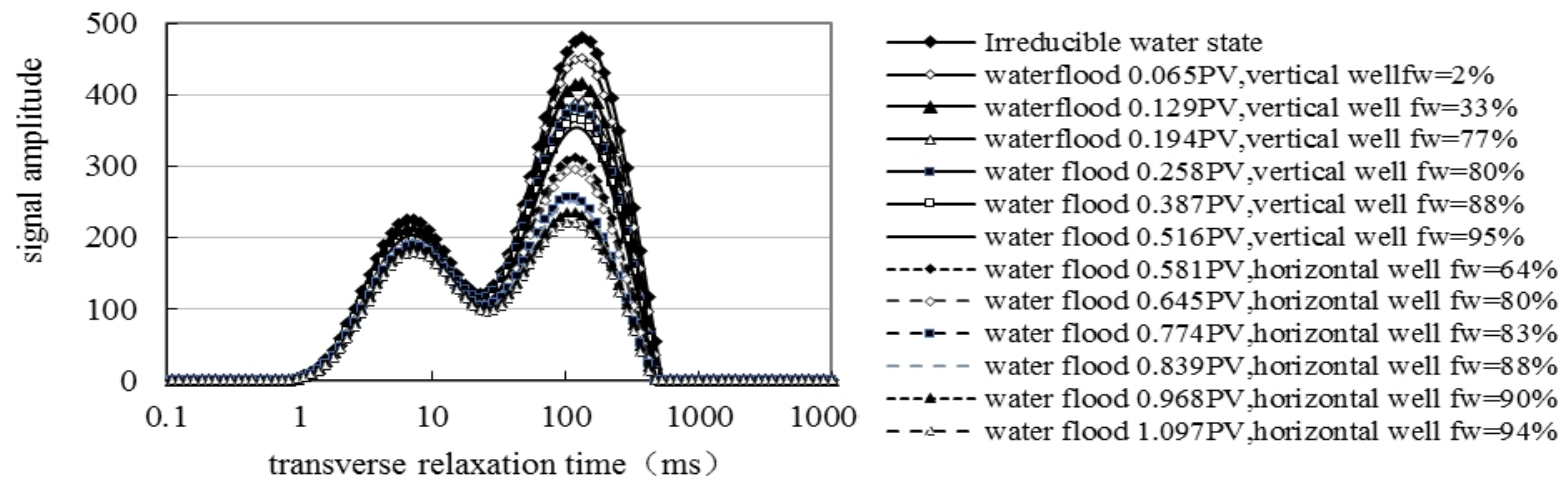

Fig.2 $\mathrm{T}_{2}$ Distribution for Horizontal Producer Experiment

The oil recovery of different pore size can also be calculated by envelope area of $\mathrm{T}_{2}$ spectrum curve. Lots of samples were drilled from physical model after horizontal well development. The oil recovery of different pore size and different layers is shown in Figure 3 and Table 1.

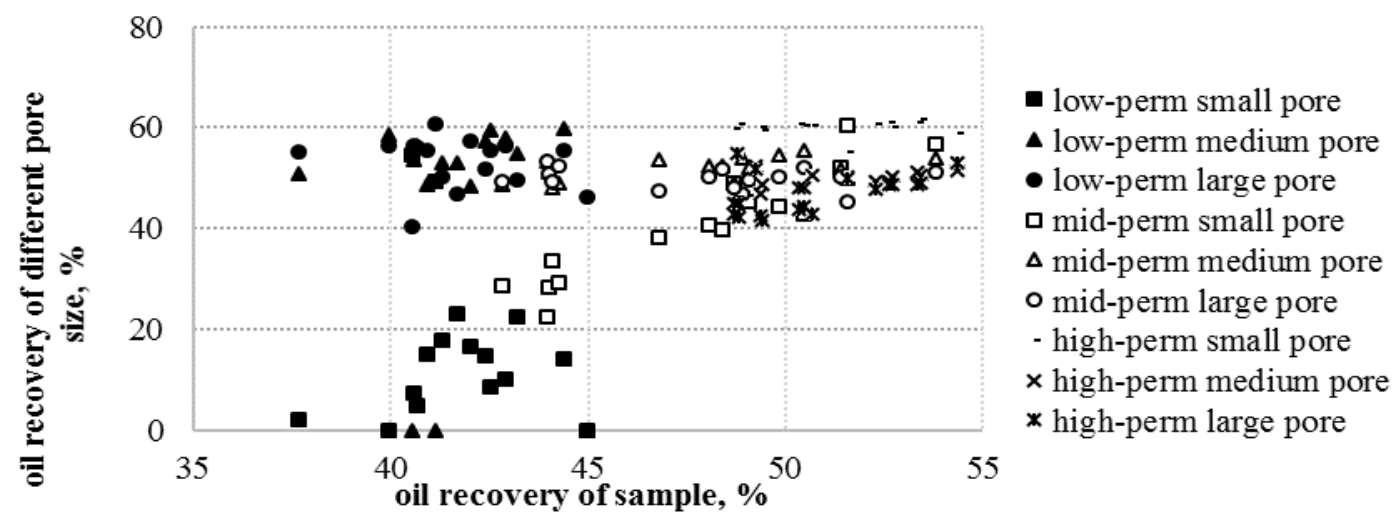

Fig. 3 Oil Recovery of Different Pore Size

Table 1 Oil Recovery of Different Pore Size and Different layers

\begin{tabular}{|c|c|c|c|}
\hline & Small pores $(<2 \mu \mathrm{m})$ & Medium pores $(2 \sim 20 \mu \mathrm{m})$ & Large pores $(>20 \mu \mathrm{m})$ \\
\hline Low-perm layer & $12.4 \%$ & $50.9 \%$ & $51.2 \%$ \\
\hline Mid-perm layer & $41.1 \%$ & $52.5 \%$ & $52.5 \%$ \\
\hline High-perm layer & $58.1 \%$ & $52.7 \%$ & $52.9 \%$ \\
\hline
\end{tabular}

The result shows that at high water cut stage $\left(\mathrm{f}_{\mathrm{w}}=95 \%\right)$, the oil recovery of large-mid pores is almost the same (the average is about $51 \%$ ), it would not change much with the sample displacement extent. However, the oil recovery of small pores changed a lot with the displacement extent of sample. This is due to the fact that the crude oil in the large-medium pore in the water flooding is preferentially displaced. When the degree of displacement is low (the oil recovery of sample is less than $40 \%$ ), oil recovery of small pores close to 0 . After further displacement (the oil recovery of 
sample is about $40 \% \sim 48 \%$ ), oil recovery of small pore increases. In the late stage of the study (the oil recovery of sample is larger than $48 \%$ ), the average oil recovery of small pores is about $58 \%$, which is higher than the average oil recovery of large-medium pores (about 52\% in this experiment). This is because after a long period of water flooding, the small pores inhale formation water through the role of infiltration as capillary, and the oil is replaced. Thus the oil recovery of small pore is higher than the average oil recovery of large-medium pores. We can also see that the remaining oil is still enriched in the low permeability layer and in the small-medium pore of mid permeability layer.

\section{Conclusions}

The crude oil in the large-medium pores in the water flooding is preferentially displaced. The ultimate recovery of large pore in both top and bottom formation is almost the same.

When the reservoir is developed to a certain extent (oil recovery in the experiment was 40\%), the crude oil in the small pores starts to be moved. With further development, the average oil recovery of small pores is even higher than the average oil recovery of large-medium pores due to infiltration. The ultimate recovery of small pores varies with the degree of displacement.

After long-term displacement, substantial amount of remaining oil exists in the low permeability layer and in the small-medium pore of mid permeability layer.

\section{Acknowledgements}

This work was financially supported by China National Offshore Oil Corporation YXKY2017ZY11.

\section{References}

[1] Roger M. Slatt. (2006). Handbook of Petroleum Exploration and Production. Volume 6: 203-248.

[2] Zao M, Guo Z, Qing H, et al. Identification of thin rock cast section and application of microscopic image analysis technology. West-China Explor Eng. Vol.3,66-70(2009).

[3] Wang Y, Zhao F, Bai B, et al. Optimized surfactant IFT and polymer viscosity for surfactant-polymer flooding in heterogeneous formations. In: SPE improved oil recovery symposium, 24-8 April, Tulsa, Oklahoma, (2010).

[4] Liu H. Study of meso-structure and damage mechanical characteristics of frozen rock based on CT image processing. Xi' an University of Science and Technology. (2013).

[5] Li H, Liu Q, Wen C, et al. Residual oil distribution and potential tapping study. Spec Oil Gas Reserv. Vol.13(3), 8-11, (2006).

[6] Kleinberg R, Vinegar HJ. NMR properties of reservoir fluids. Log Anal. Vol. 37(6):20-32, (1996).

[7] Gleeson JW, Woessner DE, Jordan CF Jr. NMR imaging of pore structures in limestones. SPE Formation Evalu. Vol.8(2),123-127, (1993).

[8] Wang W, Guo H, Ye C. Experimental studies of NMR properties of continental sedimentary rocks. Chin J Magn Reson. 2001;18(2):113-21.

[9] Kleinberg R, Vinegar HJ. NMR properties of reservoir fluids. Log Anal. 37(6):20-32(1996).

[10] Ausbrooks R, Hurley NF, May A, et al. Pore-size distribution in vuggy carbonates from core images, NMR, and capillary pressure. In: SPE annual technical conference and exhibition, 3-6 October, Houston, Texas; 1999. doi:10.2118/56506-MS. 